\title{
Effects of Subcutaneous LPS Injection on Gestational Length and Intrauterine and Neonatal Mortality in Mice
}

\author{
Yara Pujol Lopez ${ }^{a}$ Harry W.M. Steinbusch ${ }^{a}$ Bart Rutten ${ }^{a}$ Gunter Kenis ${ }^{a}$ \\ Daniel L. van den Hove ${ }^{a, b}$ Aye M. Myint ${ }^{a, c}$ \\ aschool for Mental Health and Neuroscience (MHeNS), Department of Psychiatry and Neuropsychology, Maastricht \\ University Medical Centre+, Maastricht, The Netherlands; ${ }^{b}$ Laboratory of Translational Neuroscience, Department \\ of Psychiatry, Psychosomatics and Psychotherapy, University of Würzburg, Würzburg, and 'Psychiatric Hospital, \\ Ludwig Maximilian University, Munich, Germany
}

\section{Key Words}

Infection · Pregnancy · Mortality · Mouse

\begin{abstract}
Background: Infection during pregnancy can predispose offspring to develop various psychiatric disorders such as depression in later life. In order to investigate the potential mechanisms underlying these associations, animal models of maternal infection have been employed. As such, lipopolysaccharide (LPS) has been commonly used to mimic a bacterial infection in pregnant mice. Objective: The original aim of our study was to investigate the effects of different doses of subcutaneous LPS administration on affective behavior in adult mouse offspring. In the present paper, however, we report that subcutaneous LPS administration has a profound impact on gestational length, litter size, and perinatal mortality in the offspring, even at a relatively low dose. Methods: Pregnant mice were randomly divided into 3 groups, receiving either a high $(2 \mathrm{mg} / \mathrm{kg})$ or a low $(0.5 \mathrm{mg} /$ $\mathrm{kg}$ ) dose of LPS or phosphate-buffered saline by means of subcutaneous injection. Subsequently, the effects on gesta-
\end{abstract}

(c) 2015 S. Karger AG, Basel

$1021-7401 / 15 / 0224-0274 \$ 39.50 / 0$ tional length, litter size, and perinatal mortality in the offspring were assessed. Results: After subcutaneous injection with a high dose of LPS, we observed a significant decrease in gestational length and an increase in neonatal mortality. When the low dose was administered, a tendency towards a reduced litter size was observed, most likely reflecting increased intrauterine mortality in response to prenatal maternal LPS exposure. Conclusions: We showed that subcutaneous administration of $2 \mathrm{mg} / \mathrm{kg}$ LPS to pregnant mice in the last phase of gestation should be avoided because of high offspring mortality rates, whereas subcutaneous injection of $0.5 \mathrm{mg} / \mathrm{kg}$ LPS seems to result in reabsorption of the fetuses.

๑) 2015 S. Karger AG, Basel

\section{Introduction}

Infection during pregnancy has been reported to increase the susceptibility of the offspring to developing psychopathology later in life $[1,2]$. For instance, increased risks of schizophrenia, bipolar disorder, and autism have been reported in the offspring of women infected with

\section{KARGER 125}

E-Mail karger@karger.com

www.karger.com/nim 
viruses or bacteria during pregnancy [3-5]. In order to investigate the potential mechanisms underlying these associations, animal models of maternal infection have been employed. As such, lipopolysaccharide (LPS) has been commonly used to mimic a bacterial infection in pregnant mice. Through Toll-like receptor 4 (TLR4), LPS increases the production of proinflammatory cytokines, which are known to interfere with the development of the central nervous system [6]. In the fetal brain, cytokines are known to regulate the function and development of neurons, being involved in neuroprotective and neurodegenerative processes [7]. Different effects of LPS in rodents have been reported, such as modulation of the hypothalamus-pituitary-adrenal axis, resulting in increased corticosterone levels [8]. After LPS exposure during gestation, several behavioral changes have been observed in the offspring in adulthood (e.g. impairments in spatial learning and memory) [9-11].

Intraperitoneal (i.p.) injection of LPS in pregnant mothers is known to result in fetal death depending on the dose administered [12]. In addition to the dose injected, the time point of LPS injection plays an important role in relation to changes in the protein levels of cytokines in the brains of fetuses [13]. It is noteworthy that the great majority of prenatal LPS studies have used i.p. injections in pregnant mice [12-16]. Considering the fact that i.p. injections have a failure rate in the order of $10-20 \%$ [17], subcutaneous (s.c.) injections may be favored - particularly when dealing with pregnant animals, where misplacement of the injection can cause severe damage to both the mother and the fetus. However, little is known about the potentially adverse effects of s.c. LPS injections in pregnant animals. Whereas originally the aim of our study was to investigate the effects of different doses of s.c. LPS on affective behavior in adult offspring, in the present paper we report that s.c. LPS administration, even at a relatively low dose, has a profound impact on gestational length, litter size, and perinatal mortality in the offspring.

\section{Material and Methods}

\section{Animals}

C57BL/6JRccHsd mice were obtained from Harlan Laboratories (Eystrup, Germany). The mice were housed 4 per cage in single-sex groups in individually ventilated cages sized $480 \times 375 \times$ $210 \mathrm{~mm}$ (depth $\times$ width $\times$ height) under specified pathogen-free conditions and maintained on a 12-/12-hour light-dark cycle (lights on at 12.00 a.m.) and in a temperature-controlled environment (relative humidity: $55 \pm 5 \%$; temperature: $22 \pm 2{ }^{\circ} \mathrm{C}$, and room air exchange rate: 15). The mice were s.c. injected with a transponder for identification purposes. Breeding food (Ssniff, Germany) and water were allowed ad libitum. Cages were changed every week. The experiments were conducted with permission from the government and the veterinarian administration of Upper Bavaria (AZ: 55.2-1-54-2531-61-10). After arriving at the local animal facilities, the mice were allowed to habituate to their new environment for 3 weeks. During this period, the mice were chipped to facilitate their identification. For mating, females were housed together with a male mouse for 3 days ( 1 male and 1 female per cage). Afterwards, females were housed individually. Three weeks after delivery, the offspring were weaned and separated by sex (4 mice/cage). The offspring were chipped when they were 6 weeks old.

\section{Mating and Induction of Prenatal Infection}

Approximately 8 weeks after birth, male and female mice were coupled again for 3 days. On gestational day 17, pregnancy was confirmed by visual inspection of the dam in combination with weight gain analysis (increase between the day of mating and the day of injection). Pregnant mice were randomly divided into 3 groups [high dose of LPS $(n=21)$, low dose of LPS $(n=$ $16)$, and phosphate-buffered saline $(n=20)]$. Dams were s.c. injected with 0.5 or $2 \mathrm{mg} / \mathrm{kg}$ LPS (from Salmonella enterica serotype enteritidis; Sigma Aldrich, Germany) or an equivalent volume of phosphate-buffered saline. Following injection, the mice were observed daily until delivery. Postnatal mortality was calculated by comparing the number of born pups found dead and the total number of pups counted during the observation period. Of note, when assessing postnatal mortality, 4 animals were excluded in the group treated with a low dose of LPS as these 4 animals were confirmed to be pregnant on gestational day 17 but never delivered.

\section{Statistical Analysis}

To define statistically significant differences in gestational length, litter size, and mortality, data were first subjected to an analysis of variance (ANOVA) using IBM SPSS Statistics 20 (SPSS Inc., USA). Significant overall effects were subsequently examined in more detail using post hoc Bonferroni tests. $\mathrm{p}<0.05$ was considered statistically significant in all cases.

\section{Results}

Overall, significant effects of LPS administration were found for gestational length $\left(\mathrm{F}_{2,54}=26.366 ; \mathrm{p}<0.001\right)$ and neonatal mortality $\left(\mathrm{F}_{2,50}=93.997 ; \mathrm{p}<0.001\right)$, while a trend towards a significant effect on litter size $\left(\mathrm{F}_{2,54}=\right.$ $2.700 ; \mathrm{p}=0.076$ ) was observed (fig. 1). Already 1 day after injection, some dams treated with the high dose of LPS started to deliver. This led to a significant decrease in gestational length in mothers treated with the high dose of LPS compared to the control group ( $\mathrm{p}<0.001)$, while no difference in gestational length was observed between dams injected with the low dose of LPS and the control 


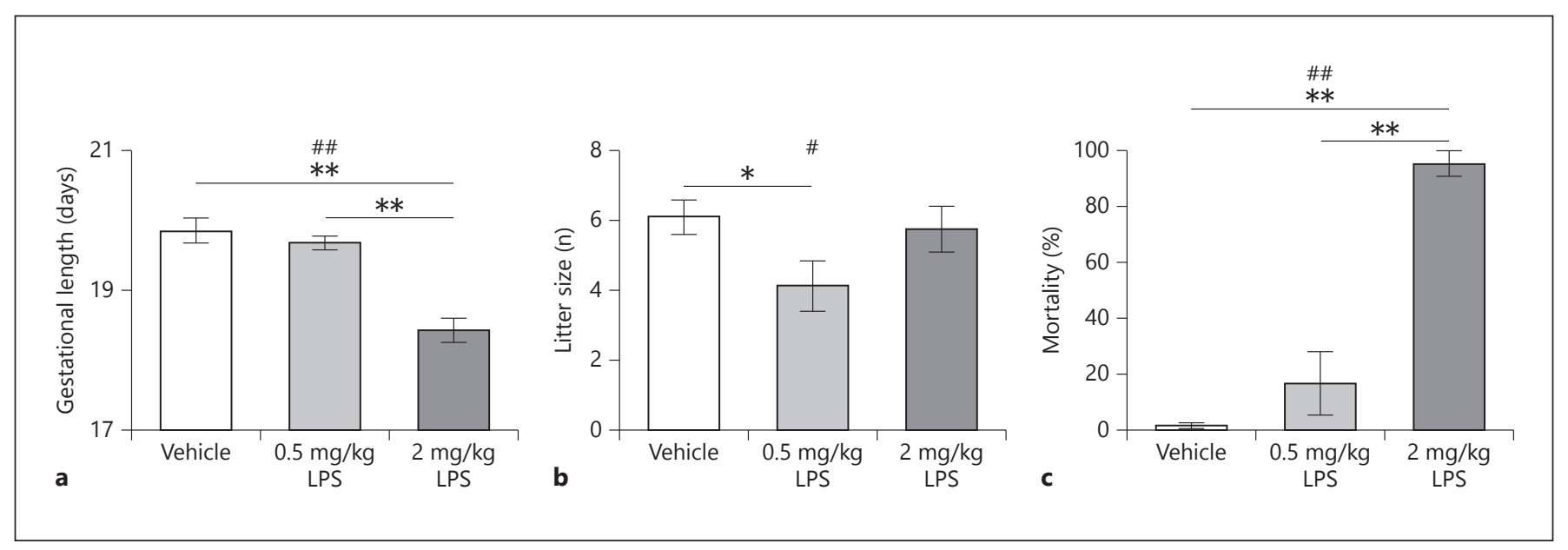

Fig. 1. Overall, significant LPS effects were found on gestational length $\left(\mathrm{F}_{2,54}=26.366 ; \mathrm{p}<0.001\right)$ and mortality $\left(\mathrm{F}_{2,50}=93.997 ; \mathrm{p}<\right.$ 0.001 ), while a trend towards a significant effect on litter size $\left(F_{2,54}=2.700 ; p=0.076\right)$ was observed. a More specifically, a reduced gestational length was observed after s.c. administration of a high dose of LPS compared to controls $(\mathrm{p}<0.001)$. A similar effect was found when analyzing both doses of LPS $(p<0.001)$. $\mathbf{b}$ The

dams $(\mathrm{p}=1)$. LPS mothers given the high dose showed a reduction in gestational length compared to those injected with a low dose $(\mathrm{p}<0.001)$. In addition, the litter size tended to be reduced in dams injected with a low dose of LPS compared to the controls, although this difference did not reach statistical significance $(p=0.095)$. Further, no significant differences were observed between the high dose of LPS and the controls $(\mathrm{p}=1)$ or between the two doses of LPS $(p=0.211)$. A remarkable effect was observed in relation to neonatal mortality. After injection of the high dose of LPS, almost all pups died within a few days after birth ( $>95 \%)$, whereas the mortality in the control offspring was almost negligible $(1.5 \%$; $\mathrm{p}<0.001)$. A mortality rate of $16.67 \%$ was detected following administration of the low dose of LPS, but this effect did not reach significance compared to the controls $(\mathrm{p}=0.233)$. When comparing both doses of LPS, a significant increase in mortality was observed in pups from mothers injected with a high dose of LPS $(\mathrm{p}<0.001)$.

\section{Discussion}

In the present paper, we report that s.c. LPS administration in the mouse has a profound impact on gestational length, litter size, and perinatal mortality in the offspring, even at a relatively low dose. More specifically, our litter size tended to be reduced after treatment with a low dose of LPS ( $\mathrm{p}=0.095)$ compared to controls. c After s.c. injection with the high dose of LPS, neonatal mortality was increased compared to values for both the low dose of LPS $(\mathrm{p}<0.001)$ and the controls ( $p<0.001)$. Bars represent means \pm SE. ${ }^{\#} p<0.1,{ }^{\# \#} p<0.001$ (ANOVA); ${ }^{*} \mathrm{p}<0.1,{ }^{* *} \mathrm{p}<0.001$ (Bonferroni).

study showed that s.c. administration of LPS at a dose of $2 \mathrm{mg} / \mathrm{kg}$ in pregnant mice resulted in a significant reduction in gestational length and a high neonatal mortality rate, whereas s.c. injection of $0.5 \mathrm{mg} / \mathrm{kg}$ LPS seemed to be associated with an increased prenatal mortality, reflecting reabsorption of the fetuses.

\section{Route of Administration}

Whereas other publications have reported similar effects after prenatal LPS administration, the exact effects seem to largely depend on the dose, the route of administration, and the gestational day of exposure or on the combination of these factors $[18,19]$. Most of the studies have used i.p. administration of LPS [e.g. 14, 16], while little is known about other ways of administration such as intrauterine or intravenous injection [20,21]. Importantly, between 10 and $20 \%$ of regular i.p. injections were placed not in the peritoneal cavity but rather in the intestine or the stomach and this frequency could not be minimized after modification of the procedure [17, 22, 23]. In fact, in the case of injection of pregnant dams, such adverse effects may be expected to be even worse, including direct harm to the fetus. In addition, other disadvantages such as splenic trauma, serosal hemorrhage, and contamination have been reported [24]. Hence, s.c. injection may represent a more suitable route of administration when dealing with pregnant mice. 


\section{Dose-Dependent Effect on Prenatal and Neonatal Mortality}

As mentioned above, the dose of LPS and the timing of the injection are considered critical factors that may impact fetal death. However, to date, no report has indicated which dose of s.c. LPS is sufficient to trigger a response in the mother and fetus without causing death. A limited number of articles have been published studying the effects of zinc supplementation and teratogenicity after prenatal exposure to LPS [25-27]. However, no evidence has been reported on effects on intrauterine or neonatal mortality, litter size, or gestational length. In the present study, the litter size tended to be reduced when using a low dose of LPS. In fact, 4 females within this group were confirmed to be pregnant at the time of LPS injection (gestational day 17) but never delivered, pointing to reabsorption of the fetuses. Interestingly, it has been reported that prenatal maternal i.p. administration of high doses of LPS causes fetal resorption, whereas exposure to low doses results in normal delivery [13]. Counterintuitively, we only observed a reduction in litter size after prenatal administration of a low dose of LPS but not after using a high dose. This lack of an effect when using the high dose could be explained by the fact that the high dose of LPS induced an abrupt premature delivery, concomitant with a massive increase in neonatal - instead of prenatal - mortality, allowing no opportunity for intrauterine absorption. Although it was expected that some pups would die after exposure to LPS, the high rate of neonatal mortality ( $>95 \%)$ observed after administration of the high dose of LPS compared to the low dose $(16.67 \%)$ was remarkable.

\section{Concluding Remarks}

Evidently, it would be useful to find out more about the exact effects of prenatal LPS administration in pups when using different doses, routes, and time points of administration in order to choose a suitable exposure for future experiments. Although most problems are to be expected when using i.p. injections, this route of administration is still mostly employed in mouse models. Therefore, we investigated the effects of different doses of LPS using s.c. injections. In our study, using s.c. LPS injections we could avoid the problem of misplacing the agent or directly harming the fetus when injecting the pregnant dams. Altogether, we conclude that s.c. LPS administration in the mouse had a profound impact on gestational length, litter size, and perinatal mortality in the offspring, even at a relatively low dose. Future work should elucidate to which extent lower doses of LPS induce short- and/or long-term behavioral changes in the offspring and which exact molecular mechanisms are involved.

\section{Acknowledgments}

This study was supported by the Commission of European Communities 7th Framework Programme collaborative project MOODINFLAME (grant No. 22963).

The authors would like to thank Andreas Weiss, Andrea Millet, and Prof. Markus Schwarz for their guidance and help in the performance of the experiments. They were employed by Ludwig Maximilian University, Munich, Germany, with financial compensation. We also thank Jo Stevens for statistical advice.

\section{References}

1 Patterson PH: Maternal infection: window on neuroimmune interactions in fetal brain development and mental illness. Curr Opin Neurobiol 2002;12:115-118.

-2 Meyer U, Yee BK, Feldon J: The neurodevelopmental impact of prenatal infections at different times of pregnancy: the earlier the worse? Neuroscientist 2007;13:241-256.

3 Brown AS: Prenatal infection as a risk factor for schizophrenia. Schizophr Bull 2006;32: 200-202.

4 Parboosing R, et al: Gestational influenza and bipolar disorder in adult offspring. JAMA Psychiatry 2013;70:677-685.

5 Atladottir $\mathrm{HO}$, et al: Maternal infection requiring hospitalization during pregnancy and autism spectrum disorders. J Autism Dev Disord 2010;40:1423-1430.
6 Boksa P: Effects of prenatal infection on brain development and behavior: a review of findings from animal models. Brain Behav Immun 2010;24:881-897.

7 Licinio J: Central nervous system cytokines and their relevance for neurotoxicity and apoptosis. J Neural Transm Suppl 1997;49: 169-175.

8 Grinevich V, et al: Effect of repeated lipopolysaccharide administration on tissue cytokine expression and hypothalamic-pituitary-adrenal axis activity in rats. J Neuroendocrinol 2001;13:711-723.

9 Lante F, et al: Late N-acetylcysteine treatment prevents the deficits induced in the offspring of dams exposed to an immune stress during gestation. Hippocampus 2008; 18 : 602-609.
10 Hao LY, et al: Prenatal exposure to lipopolysaccharide results in cognitive deficits in ageincreasing offspring rats. Neuroscience 2010; 166:763-770.

11 Golan HM, et al: Specific neurodevelopmental damage in mice offspring following maternal inflammation during pregnancy. Neuropharmacology 2005;48:903-917.

12 Renaud SJ, et al: Spontaneous pregnancy loss mediated by abnormal maternal inflammation in rats is linked to deficient uteroplacental perfusion. J Immunol 2011;186:17991808.

13 Chlodzinska N, et al: Lipopolysaccharide injected to pregnant mice affects behavior of their offspring in adulthood. Acta Neurobiol Exp (Wars) 2011;71:519-527. 
14 Xu DX, et al: Tumor necrosis factor alpha partially contributes to lipopolysaccharide-induced intra-uterine fetal growth restriction and skeletal development retardation in mice. Toxicol Lett 2006;163:20-29.

15 Buhimschi IA, Buhimschi CS, Weiner CP. Protective effect of $\mathrm{N}$-acetylcysteine against fetal death and preterm labor induced by maternal inflammation. Am J Obstet Gynecol 2003;188:203-208.

16 Salminen A, et al: Maternal endotoxin-induced preterm birth in mice: fetal responses in Toll-like receptors, collectins, and cytokines. Pediatr Res 2008;63:280-286.

17 Gaines Das R, North D: Implications of experimental technique for analysis and interpretation of data from animal experiments: outliers and increased variability resulting from failure of intraperitoneal injection procedures. Lab Anim 2007;41:312-320.
18 Mijovic JE, et al: Tyrphostins inhibit lipopolysaccharide induced preterm labor in mice. J Perinat Med 2002;30:297-300.

19 Nakamura A, et al: Increased susceptibility to LPS-induced endotoxin shock in secretory leukoprotease inhibitor (SLPI)-deficient mice. J Exp Med 2003;197:669-674.

20 Wang X, et al: Effects of intrauterine inflammation on the developing mouse brain. Brain Res 2007;1144:180-185.

21 Kohmura Y, et al: Lipopolysaccharide (LPS)induced intra-uterine fetal death (IUFD) in mice is principally due to maternal cause but not fetal sensitivity to LPS. Microbiol Immunol 2000;44:897-904.

22 Steward JP, et al: Errors in the technique of intraperitoneal injection of mice. Appl Microbiol 1968;16:1418.
23 Miner NA, Koehler J, Greenaway L: Intraperitoneal injection of mice. Appl Microbiol 1969;17:250-251.

24 Cho YJ, et al: Kinetics of proinflammatory cytokines after intraperitoneal injection of tribromoethanol and a tribromoethanol/xylazine combination in ICR mice. Lab Anim Res 2011;27:197-203.

25 Carey LC, et al: Zinc treatment prevents lipopolysaccharide-induced teratogenicity in mice. Birth Defects Res A Clin Mol Teratol 2003;67:240-245.

26 Chua JS, Rofe AM, Coyle P: Dietary zinc supplementation ameliorates LPS-induced teratogenicity in mice. Pediatr Res 2006;59:355358.

27 Chua JS, et al: Prenatal exposure to lipopolysaccharide results in neurodevelopmental damage that is ameliorated by zinc in mice. Brain Behav Immun 2012;26:326-336.

\section{Erratum}

In the article by Nascimento AF, Alves GJ, Massoco CO, Teodorov E, Felicio LF and Bernardi MM, entitled 'Lipopolysaccharide-Induced Sickness Behavior in Lactating Rats Decreases Ultrasonic Vocalizations and Exacerbates Immune System Activity in Male Offspring' [Neuroimmunomodulation 2015;22, DOI: 10.1159/000363350], an error occurred in table 1 and in the text.

Please find below the corrected table row and text passage.

\begin{tabular}{lllll}
\hline Parameter & Groups & \multirow{2}{*}{$\mathrm{p}$} \\
\cline { 2 - 3 } & control & experimental & \\
\hline $\begin{array}{l}\text { Maternal behavior } \\
\text { Latency to retrieve of 3rd pup, s }\end{array}$ & $395.26 \pm 27.27$ & $191.80 \pm 56.10$ & 0.001 \\
\hline
\end{tabular}

Section Discussion, page 6, second column, second paragraph, line 7 from the bottom: Therefore, the decrease in the latency to retrieve the third pup observed in lactating LPStreated rats in the present study may be attributable to a slight improvement in maternal care.

\section{Acknowledgement}

L.F.F. was supported by FAPESP (Grant \#2013/01610-7). 